Background Correct use of an age-appropriate restraint reduces the risk of death and injury among child passengers. While rates of age-appropriate restraint continue to rise in most developed countries, misuse of restraints remains a significant problem. Measures that target the individual are effective in reducing misuse. Carers identify instructions supplied by manufacturers as a common source of information yet there is no evidence base for developing effective instructions for targeting misuse. We aim to develop enhanced product information for child restraints through expert consensus supplemented by consumer input and testing.

Methods A modified consensus method will be used. The consensus panel consists of experts in child safety and health literacy and industry professionals. A report synthesising health literacy, and human factors design principles combined with qualitative results from consumer focus groups will be used to construct a prototype of product information (Round 1). This prototype will be sent to panel members for review and results are synthesised (Round 2). The results are redistributed and panel members rerank preferences in light of results (Round 3). Round three is repeated until consensus is reached, at which stage the prototype is finalised and tested using consumer installation and comprehension trials (Consumer input). Consumer feedback will inform the next consensus cycle.

Results The final enhanced product information will be presented at the conference with an evaluation of the modified consensus method in developing safety information for preventing misuse.

Conclusions Child restraint product information is perhaps the most widely used and underutilised channel of communication for the correct use of child restraints. Product information that is developed using a consensus method involving consumers, child safety experts and industry professionals will ensure that the information is targeted and effective at preventing misuse.

\section{ANALYSIS OF ROAD TRAFFIC INJURIES IN MEXICAN CYCLISTS}

Victoria Muro-Báez, Ma Eulalia Mendoza-García, Ricardo Pérez-Núñez. Secretariado Técnico Del Consejo Nacional Para La Prevención De Accidentes, Secretaría De Salud México (National Council for Accident Prevention Secretariat, Ministry of Health Mexico)

\subsection{6/injuryprev-2016-042156.538}

Background Recently, different Mexican cities have implemented public policies on sustainable mobility, including the promotion of bicycle as means of transport. It is not evident to what extend policies include measures to promote road safety and thus we expect road traffic injuries (RTI) will increase in this road user. To inform these policies, we analysed RTI amongst cyclist as well as helmet use.

Methods Analysis of 4 secondary databases: preliminary mortality figures, Ministry of Health hospital discharges (50\% of all hospital discharges in the country), ER and hospital statistical registries (SIS-17), and the 2012 National Health and Nutrition Survey (ENSANUT), representative at the national level. Only ENSANUT and SIS-17 report helmet use. Except for ENSANUT information analysed is of 2014, the latest information available. Results Deaths: 138 (1\% of all RTI); 97\% male, 65\% died on the scene, mean age $=46$. Most severe injuries were in the head (68\%), thorax (11\%) and multiple regions (9\%).
Hospitalizations: 392 (1\% of RTI), $83 \%$ male, mean age $=33$. Main injury was in the head (32\%), leg (17\%) and multiple regions (12\%). SIS-17: 667 cyclists were recorded, 80\% male, mean age $=30$. Helmet use was not recorded in $71 \%$, when specified 2.6\% reported its use. Most severe injury was multiple regions (29\%), head (21\%), and leg (10\%). ENSANUT: estimated 165,348 cyclists not-fatally injured, $87 \%$ male, mean age $=25$, $\mathrm{SE}=3.6$, 9.8\% used helmet. Region most affected was leg (38\%), arm (35\%) and head (23\%). Although no helmeted cyclists reported head injuries vs $24 \%$ amongst non-helmeted, difference was not statistically significant.

Conclusions Head injuries are common among cyclists and helmet use is low. Whereas cyclist-friendly infrastructure is an effective intervention to prevent injuries in the long term, helmet use could potentially reduce the frequency and severity of head injuries in the short run while bicycle widespread as a means of transport provides "safety in numbers".

\section{DRIVER LICENSING IN ABORIGINAL AND TORRES STRAIT ISLANDER PEOPLE}

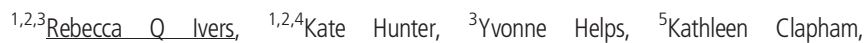
${ }^{6}$ Teresa Senserrick, ${ }^{1}$ Jake Byrne, ${ }^{2}$ Alexandra Martiniuk, ${ }^{5}$ John Daniels, ${ }^{3}$ James E Harrison. ${ }^{1}$ The George Institute for Global Health, Australia; ${ }^{2}$ The University of Sydney, Australia; ${ }^{3}$ Flinders University, Australia; ${ }^{4}$ The Poche Centre for Indigenous Health, University of Sydney, Australia; ${ }^{5}$ University of Wollongong, Australia; ${ }^{6}$ The University of NSW, Australia
\end{abstract}

\subsection{6/injuryprev-2016-042156.539}

Background Driver licensing is essential to effective road safety management systems however strengthened graduated driver licensing systems may make licensing less accessible. The impact of barriers to licensing can be far reaching, particularly for already marginalised groups. We aimed to describe licensing rates and factors associated with driver licensing for Aboriginal and Torres Strait Islander people in Australia.

Methods Interviewer administered surveys were conducted with 625 people 16 years or older in 4 Aboriginal Community Controlled Health Services in New South Wales and South Australia over a 2 week period in 2012-2013.

Results Licensing rates varied from 51\% to 77\%. A high proportion of participants reported licensing was important for independence and to meet work needs and opportunities, especially those from regional or remote locations. Employment was strongly associated with holding a current driver licence, with $64 \%$ of employed people holding a licence compared to only $36 \%$ of those who were unemployed ( $p<0.05$ ). Compared to not having a licence, having a driver licence was significantly associated with higher odds of full-time employment (OR 3.2, 95\% CI: 2.0-5.3) and educational attainment (OR 1.7, 95\% CI: 1.0-2.6 for trade or certificate; OR 3.1, 95\% CI: $1.2-8.1$ for degree qualification).

Conclusions Substantial variation in driver licensing rates across settings suggests different barriers to access. There is a strong association between driver licensing, education and employment. Licensing inequality has far-reaching impacts on the broader health and well-being of Aboriginal and Torres Strait Islander people in Australia. This study reinforces the need for appropriate and accessible pathways to achieving and maintaining driver licensing. 